\title{
Influence of Fetal Breathing Movements on Pulmonary Hemodynamics in Fetal Sheep
}

\author{
GRAEME R. POLGLASE, MEGAN J. WALLACE, DANIEL A. GRANT, AND STUART B. HOOPER
}

Fetal \& Neonatal Research Group, Department of Physiology, Monash University, Victoria 3800, Australia

\begin{abstract}
During fetal development, pulmonary vascular resistance (PVR) is high, and, as a result, blood flow through the fetal lungs is low. Although PVR markedly decreases at the time of birth, the factors that regulate pulmonary blood flow (PBF) and PVR before and immediately after birth are not clear. Our aim was to examine the relationship between episodes of fetal breathing movements (FBM) and pulmonary hemodynamics during late gestation to further understand the relationship among lung luminal volume, phasic changes in intrapulmonary pressure, and PVR before birth. In chronically catheterized fetal sheep (120128 d gestation; $n=5$; term $\sim 147 \mathrm{~d}$ ), PBF and PVR were measured during periods of FBM and apnea. Episodes of FBM were divided into periods of accentuated (amplitude of $>3.5 \mathrm{~mm}$ $\mathrm{Hg}$ change in tracheal pressure) and nonaccentuated periods of FBM. During accentuated episodes of FBM, mean PBF was increased to $159.5 \pm 23.4 \%(p<0.0025)$ of the preceding apneic period and was associated with a $19.1 \pm 5.2 \%$ reduction in PVR. In addition, during accentuated episodes of FBM, the retrograde
\end{abstract}

\section{ABSTRACT}

flow of blood through the left pulmonary artery was reduced to $90.1 \pm 1.0 \%$ of the preceding apneic period, which most likely contributed to the increase in mean PBF at this time. Although a change in PBF and PVR could not be detected during nonaccentuated FBM, compared with the preceding apneic period, PBF was linearly and positively correlated with the amplitude (change in pressure) of FBM. We conclude that PVR is decreased and PBF is increased during accentuated episodes of FBM, possibly as a result of phasic reductions in intrapulmonary pressures. (Pediatr Res 56: 932-938, 2004)
Abbreviations
FBM, fetal breathing movements
PBF, pulmonary blood flow
Pla, left atrial pressure
Ppa, pulmonary arterial pressure
PVR, pulmonary vascular resistance

At birth, blood flow through the lungs markedly increases as a result of a large reduction in pulmonary vascular resistance (PVR). Although this process plays a vital role in the transition from intra- to extrauterine life, the precise mechanisms involved are still not clear. In particular, it is not clear why PVR is high in the fetus, relative to the newborn. During fetal life, the majority $(88 \%)$ of right ventricular output bypasses the lungs and is shunted into the systemic circulation via the ductus arteriosus (1). In the late-gestation fetus, the relatively high PVR has been attributed to the low $\mathrm{PO}_{2}$ of blood perfusing the lungs $(2,3)$, to the balance between vasoconstrictor and vasodilator activity (4-7), and to the high level of resting lung expansion in the fetus (8).

Previous studies have investigated the relationship between lung luminal volume and PVR in the fetus and have shown that

Received December 2, 2003; accepted June 14, 2004

Correspondence: Graeme R. Polglase, Department of Physiology, P.O. Box, 13F, Monash University, Vic. 3800 Australia; e-mail: graeme.polglase@med.monash.edu.au This work was funded by an NHMRC of Australia project grant.

The current affiliation for D.A.G. is Ritchie Centre for Baby Health Research, Monash Institute of Reproduction and Development, Level 5, Clayton, 3168, Victoria, Australia.

DOI: 10.1203/01.PDR.0000145254.66447.C0
PVR is directly proportional to the changes in lung volume, causing pulmonary blood flow (PBF) to cease at total lung capacity (9). Conversely, a reduction in lung liquid volume, to a volume that is equivalent to the end-expiratory lung volume in the air-filled lung after birth, caused a 3- to 4-fold increase in PBF and a substantial decrease in PVR (8). Together, these studies indicate that the degree of lung expansion and the associated change in intraluminal pressure may be important physiologic determinants of PBF in the fetus, as they are in the adult. Indeed, the transmural pressure across the alveolarcapillary wall is a key factor regulating PVR in the neonatal and adult lung as capillary blood flow occurs only when capillary pressure exceeds alveolar pressure. For instance, increases in alveolar pressure in the postnatal lung, as a result of positive pressure ventilation and/or the application of continuous positive airway pressures, cause a reduction in $\mathrm{PBF}$ and a coincident decrease in cardiac output, as a result of an increase in PVR (10-13). However, the direct effects of changes in intraluminal (alveolar) pressure on PBF and PVR in the fetus are not well understood.

The intraluminal pressure changes associated with fetal breathing movements (FBM) and the role of FBM in maintain- 
ing fetal lung liquid volumes have been well documented, particularly during continuous vigorous episodes of FBM $(14,15)$. During FBM, the resistance of the upper airway to lung liquid efflux is reduced, primarily because of phasic abduction and dilation of the glottis, resulting in increased loss of liquid from the fetal lungs compared with the intervening apneic periods (15). As a result, lung liquid volumes are reduced during FBM, whereas during the intervening apneic periods, active adduction of the glottis restricts lung liquid efflux, which, therefore, tends to accumulate within the future airways. Furthermore, it is well established that FBM are usually associated with intraluminal pressure changes of 2-3 $\mathrm{mm} \mathrm{Hg}$, which can increase up to $10-15 \mathrm{~mm} \mathrm{Hg}$ during accentuated periods of FBM (14). However, it is unclear whether these FBM-induced changes in intraluminal pressure and/or lung expansion are associated with changes in PBF in the fetus. We hypothesized that accentuated or vigorous FBM are associated with an increase in PBF and a decrease in PVR compared with the intervening apneic periods. Previous studies have suggested that FBM may affect PBF and PVR, but the reported data are conflicting. Vigorous episodes of FBM, induced by a respiratory or metabolic acidosis, are reportedly associated with an increase fetal PBF (16). However, other studies have indicated that PBF is not influenced by FBM (17) and that the transient increase in $\mathrm{PBF}$ associated with the stimulation of FBM, induced by prostaglandin synthase inhibitors (meclofenamate), is not solely related to the increase in FBM (18). Our aim was to study in detail the relationship between changes in intraluminal pressure, associated with episodes of FBM, and changes in pulmonary hemodynamics in late-gestation fetal sheep.

\section{METHODS}

Aseptic surgery was performed on five pregnant ewes (Border-Leicester $\times$ Merino) at 115-118 d of gestation; gestation length is $\sim 147 \mathrm{~d}$ in this breed of sheep. Anesthesia was induced with thiopentone sodium ( $1 \mathrm{~g}$ intravenously) and was maintained, after tracheal intubation, with $1.5 \%$ halothane in $\mathrm{O}_{2}$. Antibiotics (900 mg of Depomycin, intramuscularly) were administered to the fetus immediately before any incisions were made. In each fetus, two silicone rubber cannulas (Dow Corning, cat. no. 601-365; ID $3.175 \mathrm{~mm}$, OD $6.35 \mathrm{~mm}$ ) were implanted $2-4 \mathrm{~cm}$ into the trachea, one directed toward the lungs and the other directed toward but not entering the larynx. These catheters were joined externally to form a tracheal loop that allowed the continuous flow of lung liquid. The main pulmonary artery of the fetus was exposed via a left thoracotomy through the fourth intercostal space, followed by an incision through the pericardium. The left branch of the pulmonary artery was dissected free from surrounding adventitia, and a 4-mm ultrasonic flow transducer (Transonic Systems, Ithaca, NY) was placed around it. Polyvinyl catheters (20 gauge), filled with heparinized saline, were implanted into the left pulmonary artery and into the left atrium of the fetus by direct puncture and secured in position with nonocclusive purse-string sutures. Polyvinyl catheters were also implanted into a fetal jugular vein, carotid artery, and the amniotic sac.
All catheters and the ultrasonic flow transducer cable were externalized through the right flank of the ewe. Ewes and fetuses were allowed at least $3 \mathrm{~d}$ to recover from surgery, during which time fetuses were treated daily with ampicillin (100 mg intravenously; $400 \mathrm{mg}$ intra-amniotic). Fetal arterial blood $\mathrm{PO}_{2}\left(\mathrm{PaO}_{2}\right), \mathrm{PCO}_{2}\left(\mathrm{PaCO}_{2}\right), \mathrm{pH}$, and percentage $\mathrm{O}_{2}$ saturation of $\mathrm{Hb}$ were measured every second day (ABL30; Radiometer, Copenhagen, Denmark). The flow transducer cable was attached to an internally calibrated flowmeter (Transonic Systems) for continuous measurement of flow through the left pulmonary artery. Left pulmonary arterial blood flow and left atrial, carotid, pulmonary arterial, tracheal, and amniotic sac pressures were measured using pressure transducers and recorded digitally using a data acquisition system sampling at a rate of $200 \mathrm{~Hz}$ (Powerlab; ADI, Castle Hill, Australia); mean $\mathrm{PBF}$ was calculated electronically from the instantaneous PBF signal.

Experimental protocol. Beginning on $121 \pm 1 \mathrm{~d}$ of gestation, recordings were made for $6 \mathrm{~h}$ each day for $7 \mathrm{~d}$. All episodes of FBM were recorded between $0900 \mathrm{~h}$ and $1700 \mathrm{~h}$ and commenced $\sim 1 \mathrm{~h}$ after the ewe had been fed to reduce the variability associated with circadian rhythms and the effect of maternal feeding on FBM. At the end of the seventh day, the fetal lungs were drained of liquid before the ewe and the fetus were killed by an overdose of sodium pentobarbitone administered to the ewe $(130 \mathrm{mg} / \mathrm{kg}$ intravenously). The fetus was weighed before the lungs were removed and weighed. All experimental procedures on animals were approved by the Monash University Committee for Ethics in Animal Experimentation.

Analytical methods. Each 6-h recording block was broken into episodes of FBM and apnea. Episodes of FBM were identified by negative deflections in tracheal pressure that varied in duration $(>1 \mathrm{~min})$ and were characterized into two distinct categories, accentuated and nonaccentuated FBM, on the basis of the amplitude of the deflections in tracheal pressure. Accentuated FBM were characterized by a $>3.5-\mathrm{mm} \mathrm{Hg}$ decrease in tracheal pressure, whereas nonaccentuated FBM were characterized by a $\leq 3.5-\mathrm{mm} \mathrm{Hg}$ decrease in tracheal pressure. Multiple measurements (three or fewer) were sometimes made during an FBM episode as the amplitude of the tracheal pressure fluctuations associated with FBM can vary within a single episode (Fig. 1). All measurements made within an FBM episode were compared with the period of apnea immediately preceding the episode of FBM to account for gestational age-related increases in PBF and other non-FBMspecific factors that may affect PBF. Measurements of mean $\mathrm{PBF}$ and pulse-by-pulse minimum and maximum values of PBF were electronically computed from the PBF wave form and were rapidly calculated at $\sim 2-3 \mathrm{~Hz}$, as were mean pressures in the pulmonary artery (Ppa) and left atrium (Pla). Pulmonary vascular resistance (PVR) was calculated using the formula $\mathrm{PVR}=(\mathrm{Ppa}-\mathrm{Pla}) / \mathrm{PBF}$. All transducers and the blood flowmeter were balanced and/or zeroed every day before the recordings began.

Statistical methods. A minimum of 81 measurements were obtained during periods of FBM from each fetus during the 7-d recording period; similar numbers of measurements were made 

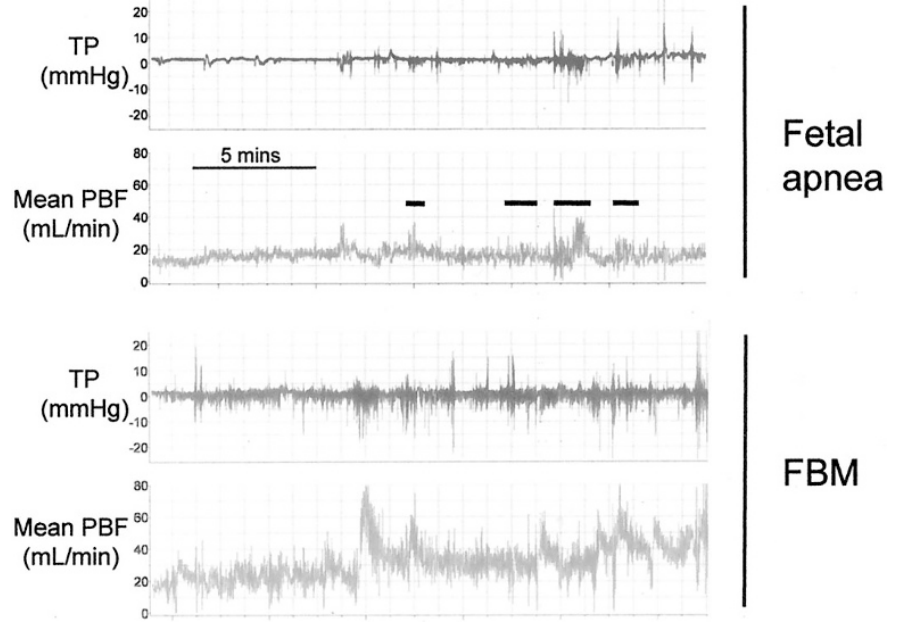

FBM

Figure 1. Recordings of tracheal pressure (TP; top traces) and mean blood flow through the left pulmonary artery (PBF; bottom traces) during a period of apnea (top) and a sustained episode of FBM (bottom) in a sheep fetus; in the bottom panel FBM are continuous. In the top panel, the bars indicate spontaneous brief episodes of FBM as depicted by negative deflections in tracheal pressure. Note that the effects of FBM on mean PBF are clearly visible but only during accentuated periods of FBM.

during accentuated and nonaccentuated periods of FBM. Each measurement represented an average value obtained from a 1to 3-min period of recording, and care was taken to avoid periods contaminated with movement or other artefacts. Measurements of PBF and PVR made during episodes of FBM were expressed as a percentage of the apneic period that immediately preceded the FBM episode. For each fetus, the mean PBF and PVR values measured during apneic periods were averaged, and then each value was expressed as a percentage of this value. All values from each fetus were then grouped, and a mean \pm SEM was calculated to provide an estimate of the normal variability of PBF and PVR that can occur between apneic periods (Fig. 2). Data in the text are expressed as the means \pm SEM. Comparisons of PBF and PVR were analyzed using paired $t$ tests. The level of significance was $p<0.05$ for all statistical analyses. For determining the relationship between the amplitude of FBM and the percentage change in PBF (as well as PVR) from the preceding apneic period, the data from each animal were divided into four separate groups depending on the FBM amplitude. For each animal, a mean value for each group was calculated and used in the regression analysis.

\section{RESULTS}

Fetal outcome. All fetuses were considered healthy according to their blood gas and acid/base status before and throughout the experiments. Measured at $126 \pm 1 \mathrm{~d}$, the mean blood gas tensions and $\mathrm{pH}$ values in blood collected from the carotid artery were as follows: $\mathrm{pH} 7.35 \pm 0.01 ; \mathrm{PaCO}_{2} 51.3 \pm 2.4 \mathrm{~mm}$ $\mathrm{Hg} ; \mathrm{PaO}_{2} 18.8 \pm 1.5 \mathrm{~mm} \mathrm{Hg}$; and percentage $\mathrm{O}_{2}$ saturation of $\mathrm{Hb} 60.8 \pm 3.0 \%$.

Pulmonary hemodynamics. An example of the recordings of tracheal pressure and mean PBF from an unanesthetized fetal sheep in utero is displayed in Fig. 1. During periods of
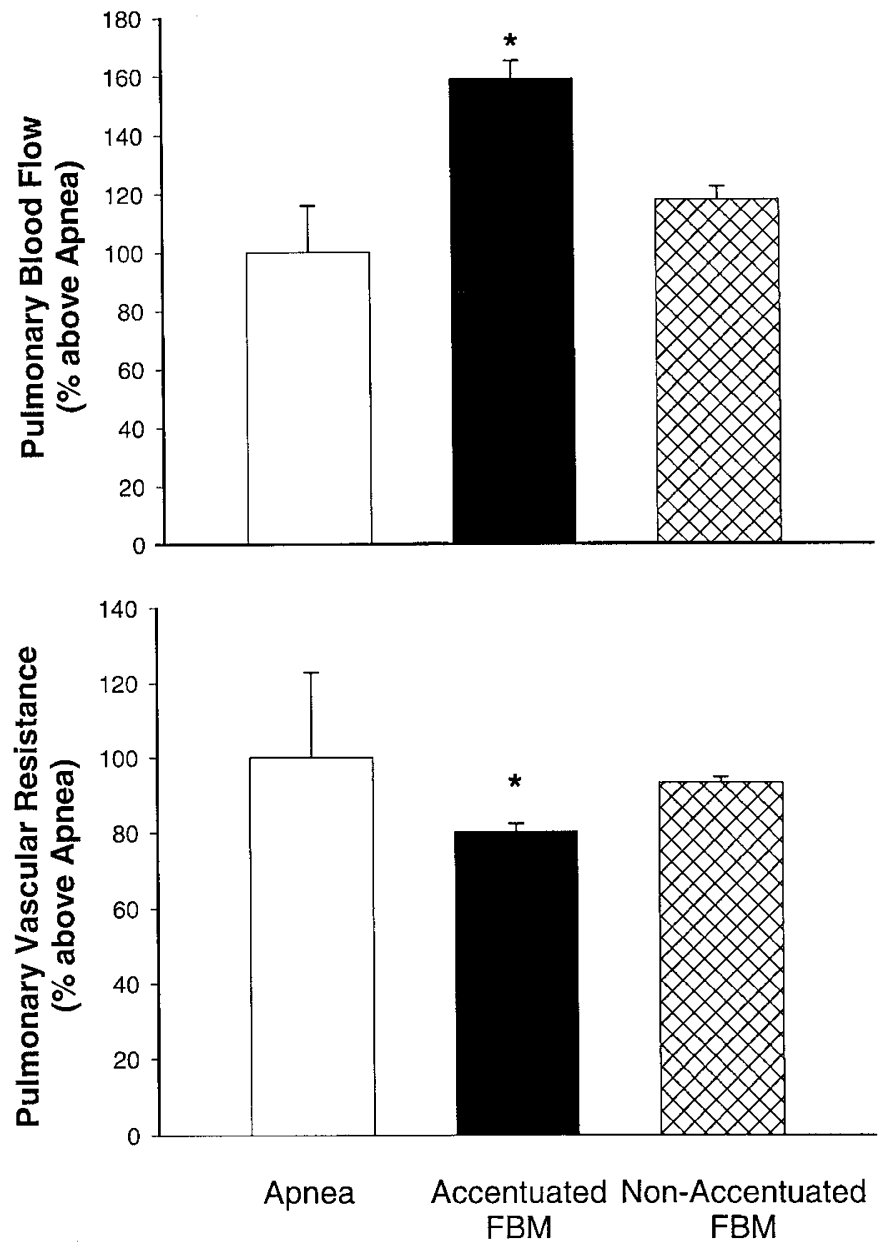

Figure 2. Mean blood flow through the left pulmonary artery $(\mathrm{PBF} ; A)$ and PVR in the left lung $(B)$, expressed as a percentage of the values measured during the preceding periods of apnea. PBF and PVR were measured during periods of apnea and accentuated and nonaccentuated periods of FBM. *Significantly different $(p<0.05)$ from the preceding apneic period $(n=5)$.

apnea, mean PBF was low, except during small epochs of FBM (Fig. 1, top). However, PBF increased markedly during episodes of accentuated FBM (Fig. 1, bottom). During episodes of accentuated FBM, when the amplitude in tracheal pressure exceeded $3.5 \mathrm{~mm} \mathrm{Hg}$, mean blood flow through the left pulmonary artery increased to $159.5 \pm 23.4 \%$ of the preceding apneic period $(100.0 \pm 16.0 \% ; p<0.025$; Fig. $2 A)$; mean PBF increased from $17.4 \pm 0.8$ to $22.6 \pm 0.9 \mathrm{~mL} / \mathrm{min}$. During nonaccentuated periods of FBM (amplitude $\leq 3.5 \mathrm{~mm} \mathrm{Hg}$ ), mean blood flow through the left pulmonary artery tended to be elevated (to $117.2 \pm 9.4 \%$ ) compared with the preceding period of apnea, although this was not significant $(p=0.078$; Fig. 2A); the mean PBF tended to increase from $20.2 \pm 0.7$ to $21.8 \pm 0.7 \mathrm{~mL} / \mathrm{min}$. Consistent with the increase in PBF during periods of accentuated FBM, PVR was significantly reduced to $80.2 \pm 5.2 \%$ of the preceding apneic period $(100.0 \pm 16.8 \%$; $p=0.015$; Fig. $2 B$ ); PVR decreased from $1.01 \pm 0.06$ to 0.96 $\pm 0.03 \mathrm{~mm} \mathrm{Hg} \cdot \mathrm{mL}^{-1} \cdot \mathrm{min}^{-1}$. During nonaccentuated periods of FBM, PVR $(92.8 \pm 3.5 \%)$ tended to be reduced compared with the preceding apneic period, although the reduction was not significant ( $p=0.058$; Fig. $2 B)$; mean PVR 
decreased from $0.85 \pm 0.07$ to $0.82 \pm 0.06 \mathrm{~mm} \mathrm{Hg} \cdot \mathrm{mL}^{-1}$. $\min ^{-1}$.

During periods of FBM, the change in PBF (from the preceding apneic period) was significantly $\left(r^{2}=0.55, p<\right.$ 0.001 ) correlated with the amplitude of FBM; this analysis included data from all fetuses and from both accentuated and nonaccentuated periods of FBM (Fig. 3A). Similarly, the change in PVR (from the preceding apneic period) was significantly $\left(r^{2}=0.59, p<0.001\right)$ but inversely correlated with the amplitude of all FBM (Fig. 3B). Furthermore, the change in PBF (from the preceding apneic period) during episodes of FBM was significantly $\left(r^{2}=0.87, p<0.001\right)$ correlated with the change in PVR (Fig. 3C).

Typical contours for blood flow through the left pulmonary artery of the fetus throughout consecutive cardiac cycles are displayed in Fig. 4. During systole, the forward flow of blood though the left pulmonary artery rapidly increases to a maxi-
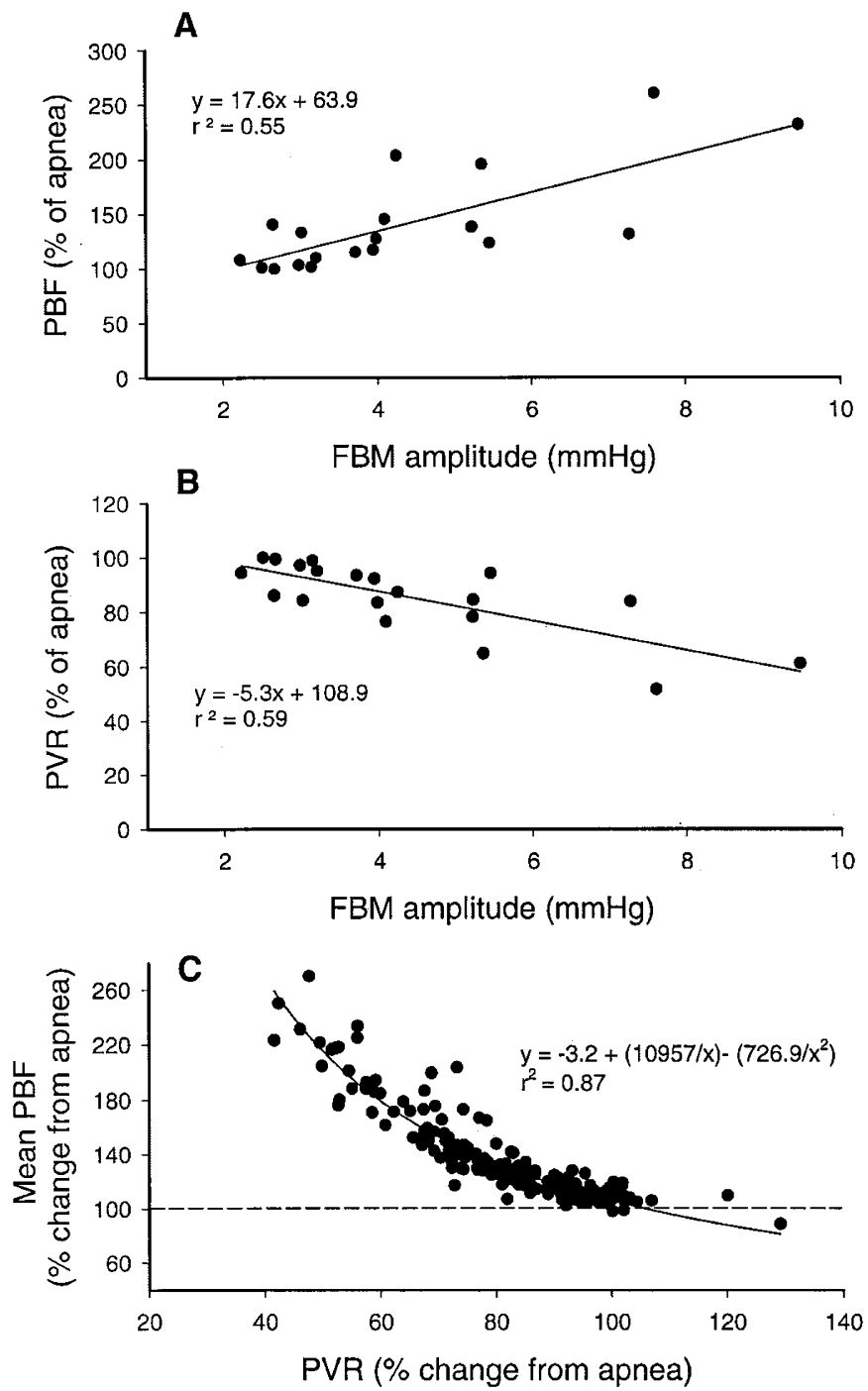

Figure 3. The relationship between mean blood flow through the left pulmonary artery $(\mathrm{PBF})$ and the amplitude in tracheal pressure $(A)$ and PVR in the left lung and the amplitude in tracheal pressure during periods of FBM (both accentuated and nonaccentuated; $n=20 ; B$ ); data obtained from five fetuses. (C) The relationship between PVR and mean PBF during episodes of FBM; data obtained from five fetuses.
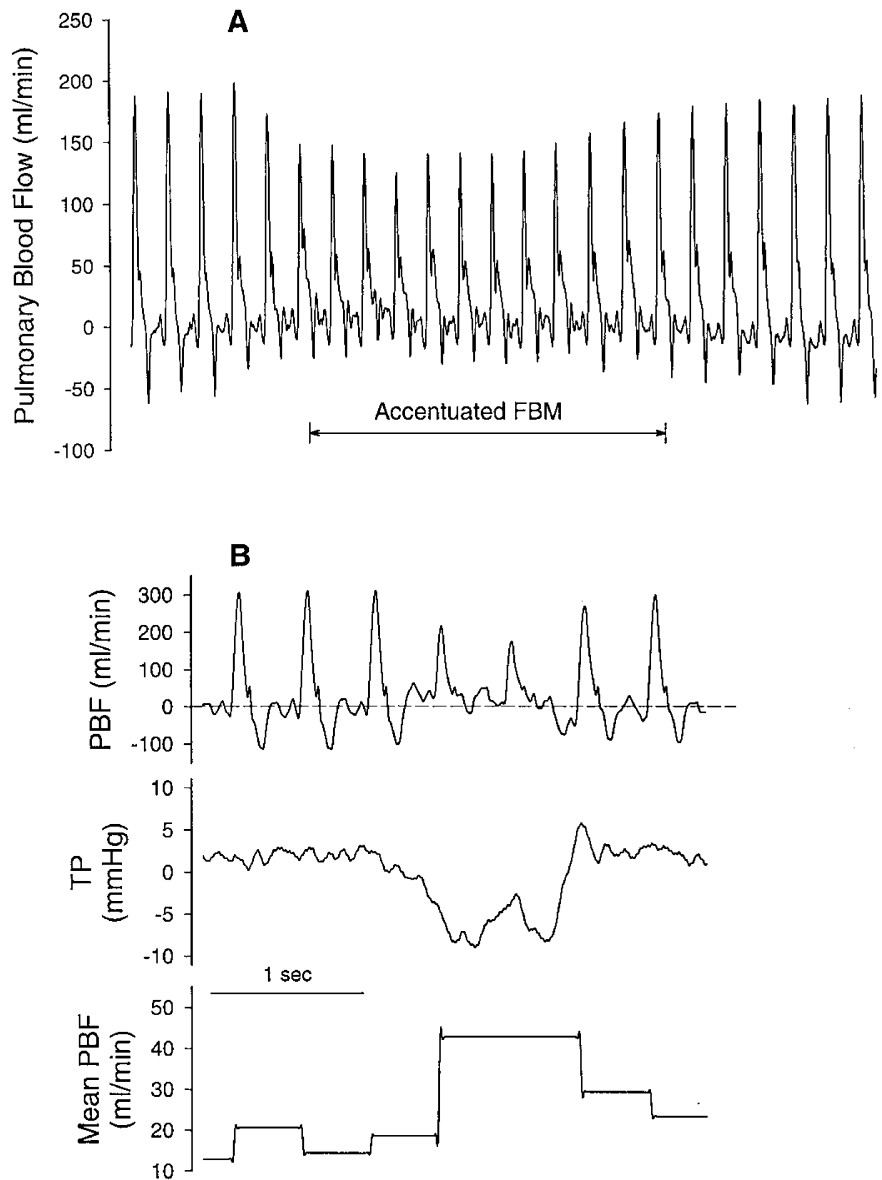

Figure 4. (A) The blood flow wave form demonstrating the instantaneous beat-by-beat flow of blood through the left pulmonary artery during normal and accentuated FBM. Forward flow in the pulmonary artery occurs only in the early part of systole and is followed by the return to zero flow. A pronounced "backflow," or retrograde flow, of blood through the left pulmonary artery occurs during diastole, which passes through the ductus arteriosus into the systemic circulation. (B) Instantaneous blood flow wave form (top recording), tracheal pressure (TP; middle recording), and mean blood flow through the left pulmonary artery (bottom recording) during a deep inspiratory effort (hiccup). Note the large increase in mean PBF and change in the left pulmonary artery blood flow wave form, which coincides with the deep inspiratory effort.

mum, then immediately declines before displaying a characteristic notch-like pattern during diastole. Eventually the flow reverses, such that blood flows retrogradely through the left pulmonary artery (as indicated by negative values). Despite the increase in mean PBF during accentuated FBM, the maximum or peak flow of blood through the left pulmonary artery was significantly reduced by $3.2 \pm 0.6 \%(p<0.05)$, compared with the preceding apneic period, during these FBM periods (Table 1, Fig. $4 A$ ); the peak flow was reduced from $311.0 \pm 8$ to 300.9 $\pm 7 \mathrm{~mL} / \mathrm{min}$. In addition, the extent of the retrograde, or backward, flow of blood through the left pulmonary artery (Table 1, Fig. 4A) was significantly reduced by $9.9 \pm 0.9 \%$ $(p<0.001)$ during episodes of accentuated FBM compared with the preceding apneic period (Table 1, Fig. 4); the retrograde flow was reduced from $-107.9 \pm 7$ to $-97.3 \pm 7$ $\mathrm{mL} / \mathrm{min}$. During nonaccentuated episodes of FBM, both the peak blood flow during systole and the retrograde (negative) flow of blood during diastole, through the left pulmonary 
Table 1. Effect of accentuated and nonaccentuated FBM on pulmonary arterial pressure, carotid arterial pressure, heart rate, and the maximum and minimum values of blood flow through the left pulmonary artery $(P B F)$ in fetal sheep at 121-128 d of gestation

\begin{tabular}{lccc}
\hline & $\begin{array}{c}\text { Nonaccentuated } \\
\text { FBM }\end{array}$ & $\begin{array}{c}\text { Accentuated } \\
\text { FBM }\end{array}$ & Apnea \\
\hline $\begin{array}{l}\text { Pulmonary arterial pressure } \\
\quad \% \text { of apnea) }\end{array}$ & $101.6 \pm 0.4$ & $104.9 \pm 0.9 *$ & $100.0 \pm 0.5$ \\
$\quad$ Carotid arterial pressure & $102.5 \pm 0.6$ & $108.5 \pm 1.9 *$ & $100.0 \pm 0.6$ \\
$\quad$ (\% of apnea) & & & \\
Heart rate (\% of apnea) & $100.9 \pm 0.3$ & $104.7 \pm 0.7 *$ & $100.0 \pm 0.5$ \\
Maximum PBF (\% of apnea) & $98.9 \pm 0.3$ & $96.8 \pm 0.6 \dagger$ & $100.0 \pm 1.7$ \\
Minimum PBF (\% of apnea) & $96.7 \pm 0.4$ & $90.1 \pm 0.9 *$ & $100.0 \pm 1.5$ \\
\hline
\end{tabular}

All values are expressed as a percentage of the preceding apneic value $(* p<0.001, \dagger p<0.05)$.

artery, were similar to that observed during the preceding apneic periods (Table 1).

Similar changes in the PBF contour that were observed during accentuated FBM were also observed during individual deep inspiratory efforts (14) (Fig. 4B). During these deep inspiratory efforts, the diaphragm is not activated in phase with the main glottic abductor muscle (19). Thus, the glottis does not dilate in phase with diaphragmatic contraction, causing a large reduction in intraluminal pressure; these efforts are not gasps and are unlikely to originate from central respiratory centers (19). Consistent with the increase in mean PBF during accentuated FBM, single deep inspiratory efforts were associated with large (2- to 3-fold) transient increases in mean PBF, which rapidly return to control values at the end of the effort (Fig. 4B). Furthermore, these deep inspiratory efforts caused marked reductions in the peak flow of blood during systole as well as reductions in the retrograde flow of blood during diastole. Indeed, some deep inspiratory efforts almost completely abolished the negative or retrograde flow of blood through the left pulmonary artery during diastole (Fig. $4 B$ )

During accentuated episodes of FBM, the fetal heart rate was significantly increased by $12.8 \pm 4.2 \mathrm{bpm}$ compared with the preceding apneic period (Table 1); the heart rate increased from $167.7 \pm 1$ to $175.2 \pm 1 \mathrm{bpm}$. However, during nonaccentuated FBM, the fetal heart rate was similar to that observed during the preceding apneic period (Table 1). Similarly, during periods of accentuated FBM, Ppa and systemic arterial pressure both were significantly increased compared with the preceding apneic period (from $31.1 \pm 0.6$ to $32.9 \pm 0.6 \mathrm{~mm} \mathrm{Hg}$ and from $29.9 \pm 1.4$ to $32.0 \pm 1.4 \mathrm{~mm} \mathrm{Hg}$, respectively) but were not altered during nonaccentuated periods of FBM (Table $1)$.

\section{DISCUSSION}

Our results indicate that mean PBF increases and PVR decreases during accentuated periods of FBM (amplitude $>3.5$ $\mathrm{mm} \mathrm{Hg}$ ) but seem to remain unaltered during episodes of nonaccentuated FBM periods. In particular, mean PBF increased by $\sim 60 \%$ during episodes of accentuated FBM, which most probably was due to the combined effect of an associated decrease in PVR (by $\sim 20 \%$ ) as well as an increase in right ventricular output leading to an increase in driving pressure; this was indicated by an increase in fetal heart rate $(\sim 13 \%)$ and pulmonary arterial pressure $(\sim 5 \%)$. Despite the decrease in PVR, during episodes of accentuated FBM, the peak forward flow of blood through the pulmonary artery during systole was reduced (by $\sim 3.3 \%$ ) as was the backward flow of blood during diastole. These results clearly indicate that during fetal life, PBF and PVR are influenced by episodes of FBM, particularly when the amplitude in tracheal pressure associated with each breathing movement exceeds $3.5 \mathrm{~mm} \mathrm{Hg}$. The resulting change in PBF is, in some cases, considerable, inducing a 5- to 6-fold increase in mean $\mathrm{PBF}$ when the change in intraluminal pressure associated with the inspiratory effort is large (Figs. 1 and 4).

Our findings extend the previously reported findings (abstract only) that mean PBF increases during stimulated FBM resulting from a respiratory or metabolic acidosis (16). However, our findings demonstrate that increases in PBF and decreases in PVR are a normal feature of fetal life when FBM become accentuated. Furthermore, our data provide important additional information as to the factors that regulate PVR before birth. In particular, our findings indicate that intraluminal pressure is likely to be an important determinant of PBF in the fetus, as it is postnatally, possibly by regulating the degree of capillary compression (9).

Previous studies $(17,18,20)$ have reported that PBF is not influenced by FBM and concluded that FBM do not stimulate lung growth by intermittent increases in PBF, although one study did report that PBF increased with the onset of PBF and was reduced upon the cessation of FBM (21). However, in those studies, the FBM were not divided into accentuated and nonaccentuated episodes, and comparisons were not made between episodes of FBM and the preceding apneic period. As the incidence of nonaccentuated FBM greatly exceeds that of accentuated FBM, it is likely that the effect of accentuated FBM on PBF was masked in the analysis by prolonged periods of low amplitude FBM $(<3 \mathrm{~mm} \mathrm{Hg})$. However, we divided the epochs of FBM into accentuated and nonaccentuated periods of FBM, as defined by us and others $(15,21,22)$, and recorded FBM for $6 \mathrm{~h} / \mathrm{d}$ for $7 \mathrm{~d}$, which allowed a rigorous analysis of the impact of FBM on PBF and PVR. Furthermore, as PBF increases with gestational age and can vary considerably between fetuses and within individual fetuses over a 24-h period, a comparison of PBF during episodes of FBM with the preceding apneic period is a sensitive method for assessing the specific effects of FBM on PBF. It is of interest that a previous study noted a transient increase in PBF within 30-90 min of infusing a prostaglandin synthase inhibitor (meclofenamate), which increases both the incidence and the amplitude of FBM (18). Although it was concluded that factors other than FBM contributed to the increase in mean PBF, the authors did speculate that "the initial increase in PBF may be in part due to the deeper fetal respirations subsequent to meclofenamate." This speculation is consistent with our finding of a strong relationship between the amplitude of FBM and mean PBF (Fig. 3A).

The increase in PBF during accentuated FBM could have resulted from the combined effect of a decrease in PVR as well as an increase in right ventricular output, leading to an increase in driving pressure. Although we did not measure right ven- 
tricular output in this study, it is likely to have been increased during accentuated FBM. This is indicated by an increase in fetal heart rate (by $12.8 \pm 4.2 \mathrm{bpm}$ ) in combination with an $\sim 5 \%$ increase in left pulmonary arterial pressure (23) and has been observed previously during accentuated FBM $(21,22)$. This finding is consistent with previous studies that demonstrated that vigorous FBM impose a significant oxygen demand in the fetus (21) and are commonly associated with increased fetal body movements (24). Indeed, during vigorous FBM, fetal oxygen consumption can increase by $30 \%$ (21), and blood flow to the main respiratory muscles (diaphragm and intercostals) increases (22).

We consider that the decrease in PVR observed during accentuated FBM was the predominant contributor to the increase in PBF during such episodes. This is because of the very close inverse exponential relationship that we observed between PVR and PBF (Fig. 3C) during FBM. The inverse exponential nature of this relationship indicates that when the mean $\mathrm{PBF}$ is equivalent to or less than that measured during apnea, PBF is near its minimum point, physiologically, and that large increases in PVR are required to reduce it further. Indeed, we commonly observed mean PBFs of $0-10 \mathrm{~mL} / \mathrm{min}$, particularly during periods of apnea (Fig. 1). This finding raises the concept that during fetal life, PVR is highest and PBF is lowest during apnea when intraluminal pressures are 1-2 $\mathrm{mm}$ $\mathrm{Hg}$ above ambient (amniotic sac) pressure (25). Then, during episodes of FBM, PVR decreases and PBF increases as a result of the phasic decrease in intraluminal pressure associated with inspiratory efforts; the extent of the changes in PVR and PBF depend on the amplitude of the change in intraluminal pressure (Fig. $3 A$ and $B$ ). The natural extension to this argument is the suggestion that the high intraluminal pressure within the fetal lung, relative to ambient pressure, is a major contributing factor to the high PVR in the fetus, particularly during periods of fetal apnea; this is in addition to vasoconstrictor activity.

Previous studies have shown that PVR is directly related to changes in fetal lung liquid volumes (9), resulting in substantial reductions in PVR (2- to 3-fold) and increases in PBF when fetal lung liquid volumes are reduced (8). As FBM are associated with increased loss of lung liquid, which can result in substantial changes in lung liquid volume (15) particularly during vigorous FBM, a reduction in lung expansion could account for the observed decrease in PVR during vigorous FBM. However, although this mechanism may contribute to the decrease in PVR during FBM and account for the large variability in PBF between epochs of FBM, it is unlikely to be the primary mechanism involved. Indeed, short periods of vigorous FBM, which were too short to produce substantial changes in lung volume (Fig. 1), as well as single deep inspiratory efforts (Fig. 4B), coincided with large increases in mean PBF. Similarly, it is unlikely that an increase in shear stress-mediated vasodilator release from endothelial cells could explain the FBM-associated decrease in PVR as the changes in mean PBF were tightly constrained to the periods of reduced intraluminal pressure (Figs. 1 and $4 B$ ). Instead, we suggest that the decrease in PVR mainly resulted from the large phasic reduction in intraluminal pressure associated with vigorous FBM. This would be expected to reduce perivascular tissue pressure and increase the alveolar/capillary transmural pressure, causing capillary expansion and the facilitation of capillary blood flow (12). Indeed, we found that the amplitude of the change in tracheal pressure associated with FBM was closely correlated with the increase in mean PBF and decrease in PVR (Fig. $3 A$ and $B$ ). More specific, we also found that single deep inspiratory efforts (analogous to hiccups), which are a normal feature of fetal respiratory development and generate large reductions in intraluminal pressure $(15,19)$, were closely associated with a transient increase (4- to 5-fold) in PBF (Fig. 4).

The changes in the beat-to-beat peak forward flow and retrograde flow through the pulmonary artery, associated with vigorous FBM, were surprising, and the mechanisms involved are unknown; these changes also occurred during spontaneous single deep inspiratory efforts. The decrease in peak forward flow usually occurred despite a coincident increase in driving pressure (as measured by the pressure difference between the left pulmonary artery and left atrium) and a decrease in PVR. One possible explanation is that if the increase in pulmonary artery pressure led to an increase in the pressure gradient across the ductus arteriosus, then a greater proportion of the highvelocity components of the pulse may have traversed the low-resistance pathway through the ductus arteriosus into the systemic circulation. As a result, the peak flow of blood passing through the major branches of the pulmonary arteries (site of measurement) would be reduced. However, the net result was not a decrease in mean PBF, because there was an even greater decrease in retrograde flow during diastole than the reduction in forward flow during systole. The backward flow of blood through the left pulmonary artery during diastole has previously been reported $(1,26)$ and is thought to be a consequence of the very high PVR in the fetus in addition to the presence of the ductus arteriosus (27). Thus, during diastole, blood is thought to reflect off the highly constricted pulmonary vascular bed and flow retrogradely along the left pulmonary artery before passing through the ductus arteriosus, into the systemic circulation (26). The reduction in backward flow through the left pulmonary artery during vigorous FBM is likely to be a consequence of the decrease in PVR (of $\sim 20.0 \%$ ) during vigorous FBM. This decrease in PVR was sufficient to reduce the backflow of blood from a peak value of -160 $\mathrm{mL} / \mathrm{min}$ during apneic periods to $-46.5 \pm 10 \mathrm{~mL} / \mathrm{min}$ during vigorous FBM with a range of values from -20 to -120 $\mathrm{mL} / \mathrm{min}$. Thus, in some circumstances, the decrease in PVR was sufficient almost to abolish the backflow of blood through the left pulmonary artery, thus allowing blood flow to continue in a forward direction throughout the cardiac cycle. The decrease in retrograde flow was particularly obvious during deep inspiratory efforts (Fig. 4A).

\section{CONCLUSION}

In summary, the findings of this study indicate that episodes of spontaneous, vigorous FBM during normal fetal development are associated with large increases in PBF. These increases in PBF are predominantly due to a reduction in PVR, although an associated increase in right ventricular output may 
contribute to the increase in PBF. Understanding the mechanisms involved could have important implications for understanding the mechanisms responsible for maintaining a high PVR in the fetus as well as understanding the factors that lead to the reduction in PVR at birth.

\section{REFERENCES}

1. Friedman AH, Fahey JT 1993 The transition from fetal to neonatal circulation: normal responses and implications for infants with heart disease. Semin Perinatol 17:106121

2. Teitel DF, Iwamoto HS, Rudolph AM 1990 Changes in the pulmonary circulation during birth-related events. Pediatr Res 27:372-378

3. Iwamoto HS, Teitel DF, Rudolph AM 1993 Effects of lung distension and spontaneous fetal breathing on hemodynamics in sheep. Pediatr Res 33:639-644

4. Cassin S 1993 The role of eicosanoids and endothelium-dependent factors in regulation of the fetal pulmonary circulation. J Lipid Mediat 6:477-485

5. Wong J, Fineman JR, Heymann MA 1994 The role of endothelin and endothelin receptor subtypes in regulation of fetal pulmonary vascular tone. Pediatr Res 35:664670

6. Heymann MA 1999 Control of the pulmonary circulation in the fetus and during the transitional period to air breathing. Eur J Obstet Gynecol Reprod Biol 84:127-132

7. Rairigh RL, Storme L, Parker TA, Le Cras TD, Markham N, Jakkula M, Abman SH 2000 Role of neuronal nitric oxide synthase in regulation of vascular and ductus arteriosus tone in the ovine fetus. Am J Physiol 278:L105-L110

8. Hooper SB 1998 Role of luminal volume changes in the increase in pulmonary blood flow at birth in sheep. Exp Physiol 83:833-842

9. Walker AM, Ritchie BC, Adamson TM, Maloney JE 1988 Effect of changing lung liquid volume on the pulmonary circulation of fetal lambs. J Appl Physiol 64:61-67

10. Rankin JS, Olsen CO, Arentzen CE, Tyson GS, Maier G, Smith PK, Hammon JW Jr, Davis JW, McHale PA, Anderson RW, Sabiston DC Jr 1982 The effects of airway pressure on cardiac function in intact dogs and man. Circulation 66:108-120

11. Fuhrman BP, Everitt J, Lock JE 1984 Cardiopulmonary effects of unilateral airway pressure changes in intact infant lambs. J Appl Physiol 56:1439-1448
12. Fuhrman BP, Smith-Wright DL, Kulik TJ, Lock JE 1986 Effects of static and fluctuating airway pressure on intact pulmonary circulation. J Appl Physiol 60:114-122

13. Fuhrman BP, Smith-Wright DL, Venkataraman S, Howland DF 1989 Pulmonary vascular resistance after cessation of positive end-expiratory pressure. J Appl Physiol 66:660-668

14. Dawes GS, Fox HE, Leduc BM, Liggins GC, Richards RT 1972 Respiratory movements and rapid eye movement sleep in the foetal lamb. J Physiol 220:119-143

15. Harding R, Dickson KA, Hooper SB 1989 Fetal breathing, tracheal fluid movement and lung growth. In: Gluckman PD, Johnston BM, Nathanielsz PW (eds) Advances in Fetal Physiology. Perinatology Press, New York, pp 153-175

16. Molteni RA, Douglas M, Jones MD Jr, Simmons MA 1980 The effect of breathing on pulmonary blood flow in the sheep fetus. Pediatr Res 14:648

17. Savich RD, Guerra FA, Lee CC, Kitterman JA 1994 The effect of fetal breathing movements on pulmonary blood flow in fetal sheep. Pediatr Res 35:484-489

18. Savich RD, Guerra FA, Lee CC, Kitterman JA 1995 Effect of inhibition of prostaglandin synthesis on breathing movements and pulmonary blood flow in fetal sheep. J Appl Physiol 78:531-538

19. Harding R 1980 State-related and developmental changes in laryngeal function. Sleep 3:307-322

20. Savich RD, Guerra FA, Lee CC, Kitterman JA 1995 Prostaglandin $E_{2}$ decreases fetal breathing movements but not pulmonary blood flow, in fetal sheep. J Appl Physiol 78:1477-1484

21. Rurak DW, Gruber NC 1983 Increased oxygen consumption associated with breathing activity in fetal lambs. J Appl Physiol 54:701-707

22. Molteni RA, Melmed MH, Sheldon RE, Jones MD, Meschia G 1980 Induction of fetal breathing by metabolic acidemia and its effect on blood flow in the respiratory muscles. Am J Obstet Gynecol 136:609-620

23. Marshall BE, Marshall C 1991 Pulmonary hypertension. In: Crystal RA, West JB (eds) The Lung. Raven Press, New York, pp 1177-1187

24. Rurak DW, Richardson BS, Patrick JE, Carmichael L, Homan J 1990 Oxygen consumption in the fetal lamb during sustained hypoxemia with progressive acidemia. Am J Physiol 258:R1108-R1115

25. Vilos GA, Liggins GC 1982 Intrathoracic pressures in fetal sheep. J Dev Physio 4:247-256

26. Rudolph AM 1977 Fetal and neonatal pulmonary circulation. Am Rev Respir Dis 115:11-18

27. Abman SH, Accurso FJ 1989 Acute effects of partial compression of ductus arteriosus on fetal pulmonary circulation. Am J Physiol 257:H626-H634 